\title{
Erratum
}

\section{The Gold(I)-Catalyzed Cycloisomerization of 1,6-Enynes to 1,4-Dienes}

Sang Ick Lee, Soo Min Kim, Sun Young Kim, Young Keun Chung* Synlett 2006, 2256.

An incorrect structure was inadvertently shown for compound $\mathbf{2}$. The correct figure is shown below. The authors regret the error and misinterpretation of the original data.

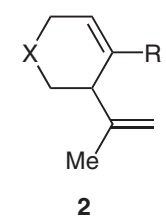

In equations 1 and 4 and Tables 1-2, 2 was replaced by the correct structure. Scheme 1 should be replaced by a new one shown below.

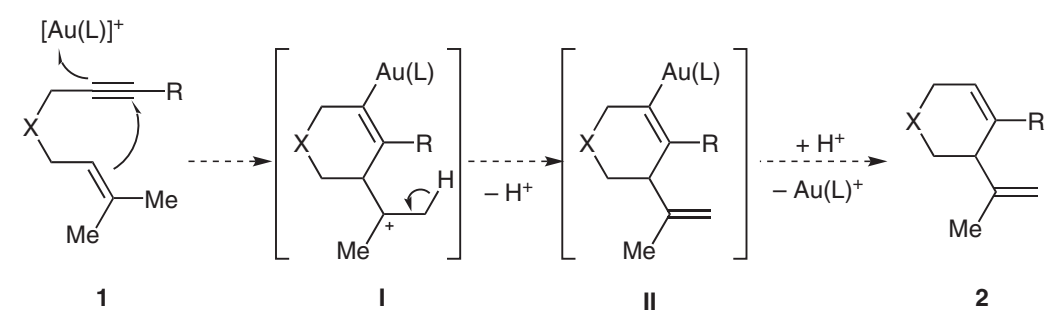

On page 2257, in Table 1, last column: 2A/3A should read 2a/3a. On the same page, right column, in the 4th and the last line from the bottom: 5-exo-dig should be deleted and E-product 2 should be changed to product 2.

In Refences and Notes section compounds $\mathbf{2 a - i}$ should be correctly named as follows:

2a: 4-Phenyl-3-(prop-1-en-2-yl)-1-tosyl-1,2,3,6-tetrahydropyridine

2b: 1-(Mesitylsulfonyl)-4-phenyl-3-(prop-1-en-2-yl)-1,2,3,6-tetrahydropyridine

2c: 4-Phenyl-3-(prop-1-en-2-yl)-3,6-dihydro- $2 H$-pyran

2d: 4-Methyl-3-(prop-1-en-2-yl)-1-tosyl-1,2,3,6-tetrahydropyridine

2e: 4-Cyclopropyl-3-(prop-1-en-2-yl)-1-tosyl-1,2,3,6-tetrahydropyridine

2f: 4-Cyclohexenyl-3-(prop-1-en-2-yl)-1-tosyl-1,2,3,6-tetrahydropyridine

2g: 4-(4-Methoxyphenyl)-3-(prop-1-en-2-yl)-1-tosyl-1,2,3,6-tetrahydropyridine

2h: 4-(Naphthalen-1-yl)-3-(prop-1-en-2-yl)-1-tosyl-1,2,3,6-tetrahydropyridine

2i: 4-(3,5-Dimethylphenyl)-3-(prop-1-en-2-yl)-1-tosyl-1,2,3,6-tetrahydropyridine 
Figure 1 should be replaced by the X-ray crystal structure shown below:

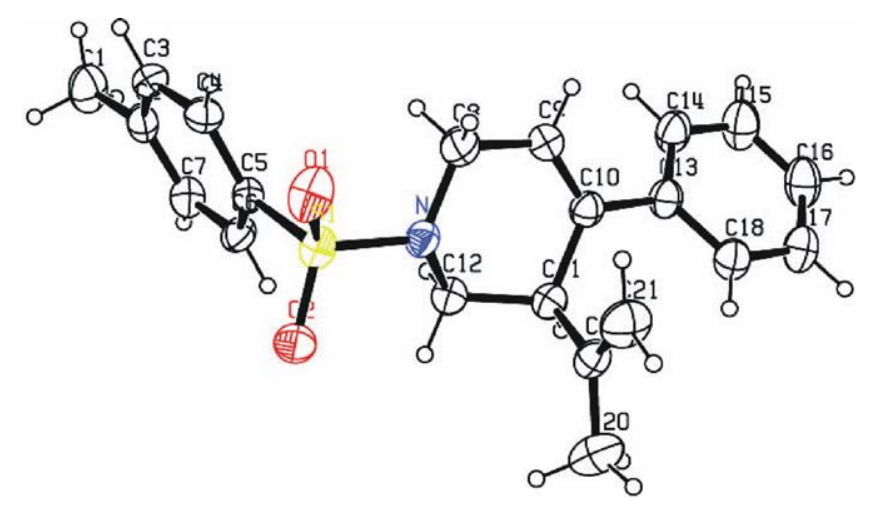

Some signals in the ${ }^{1} \mathrm{H}$ NMR data of compounds $\mathbf{1 e}, \mathbf{f}$ and $\mathbf{2 a}, \mathbf{e}$ were incorrectly assigned; the corrected values should read as follows:

1e: $7.69(\mathrm{~d}, J=8.3 \mathrm{~Hz}, 1 \mathrm{H}) \rightarrow 7.69(\mathrm{~d}, J=8.3 \mathrm{~Hz}, 2 \mathrm{H})$.

1f: $7.74(\mathrm{~d}, J=8.3 \mathrm{~Hz}, 1 \mathrm{H}) \rightarrow 7.74(\mathrm{~d}, J=8.3 \mathrm{~Hz}, 2 \mathrm{H})$.

2a: 4.78 (s, $3 \mathrm{H}), 4.88(\mathrm{~s}, 3 \mathrm{H}) \rightarrow 4.78(\mathrm{~s}, 1 \mathrm{H}), 4.88(\mathrm{~s}, 1 \mathrm{H})$.

2e: $0.45(\mathrm{~m}, 1 \mathrm{H}), 0.56(\mathrm{~m}, 1 \mathrm{H}), 1.20(\mathrm{~m}, 2 \mathrm{H}) \rightarrow 0.21(\mathrm{~m}, 1 \mathrm{H}), 0.45(\mathrm{~m}, 1 \mathrm{H}), 0.56(\mathrm{~m}, 2 \mathrm{H}), 1.20(\mathrm{~m}, 1 \mathrm{H}) ; 7.30(\mathrm{~d}$, $J=8.5 \mathrm{~Hz}, 1 \mathrm{H}), 7.64(\mathrm{~d}, J=8.3 \mathrm{~Hz}, 1 \mathrm{H}) 7.30(\mathrm{~d}, J=8.5 \mathrm{~Hz}, 2 \mathrm{H}), 7.64(\mathrm{~d}, J=8.3 \mathrm{~Hz}, 2 \mathrm{H})$.

The authors apologize for the above mistakes. 